\title{
Exchange effects in plasmas: The case of low-frequency dynamics
}

\author{
J. Zamanian, ${ }^{1, *}$ M. Marklund, ${ }^{1,2, \dagger}$ and G. Brodin ${ }^{1, \ddagger}$ \\ ${ }^{1}$ Department of Physics, Umea University, SE-901 87 Umea , Sweden \\ ${ }^{2}$ Department of Applied Physics, Division for Condensed Matter Theory, Chalmers University of Technology, SE-412 96 Göteborg, Sweden
}

(Received 14 March 2013; revised manuscript received 12 November 2013; published 11 December 2013)

\begin{abstract}
Recently, there has been a surge of interest in nonequilibrium collective quantum models, where particle dispersion and spin are examples of effects taken into account. Here, we derive a kinetic plasma model containing fermion exchange effects. Exchange interactions are of great importance in many systems and have no classical analogy. Our model therefore constitutes a possible probe of collective quantum phenomena in other regimes. As an example, we consider the influence of the exchange effect on low-frequency dynamics, in particular ionacoustic waves. Comparisons to related computational techniques are given and the differences are highlighted. Furthermore, we discuss the applicability of our model, its limitations, and possible extensions.
\end{abstract}

DOI: 10.1103/PhysRevE.88.063105

PACS number(s): 52.25.Dg

\section{INTRODUCTION}

Quantum plasma physics is currently a field of intense study. One reason for this is the potential applications in, for example, laser-produced plasmas [1-4], ultrasmall electronic devices, and dense astrophysical systems [5-7]. Different aspects of quantum plasmas have been studied such as quantum dispersion and Fermi pressure [5-7], the magnetic dipole force and the spin dynamics [8-17], quantum relativistic effects, and nonlinear dynamics [14-16,18,19]. Typically, quantum effects are important for systems with high density and low temperature. That said, it is important to distinguish between the quantum effects related to thermodynamic equilibrium properties and to dynamical properties of the system. Exchange effects due to particle statistics have been successfully included in the density functional theory (DFT) [20,21]. Applications of DFT include, for example, ground-state properties of atoms and equilibrium properties of many-particle systems $[20,21]$. The effects of exchange on dynamics have also been studied in the setting of kinetic theory [8,22-28], as well as in studying, e.g., the thermodynamic properties of plasmas [24,25]. It has also been studied using fluid theory [28]. Furthermore, many papers deal with how quantum mechanics affects the low-frequency long-scale dynamics, as, for example, quantum ion-acoustic waves [29-33].

In Sec. II, we derive the Wigner equation for electrons within the Hartree-Fock approximation. We simplify the equation by assuming that the plasma is not spin polarized and by focusing on length scales much longer than the thermal de Broglie wavelength. In Sec. III, we consider the impact on ion-acoustic waves by treating the exchange effects perturbatively within the linear approximation and, finally, in Sec. V, we discuss our result.

\section{MODEL}

Here we consider a completely ionized electron-ion plasma with the particles interacting through a mean-field scalar

\footnotetext{
*jens.zamanian@physics.umu.se

†mattias.marklund@chalmers.se

${ }^{\ddagger}$ gert.brodin@physics.umu.se
}

potential. Quantum effects for the ions will be completely neglected, while for the electrons we will take into account a dynamic correction due to the Pauli exclusion principle. Also, we will not consider effects due to the self-energy and particle correlations [25]. Here we give an outline of the derivation of a kinetic theory with exchange effects.

The state of the $N$ electrons is described by the density operator $\rho_{1 \ldots N}$ (see, for example, Ref. [25]) and the dynamics is given by the von Neumann equation with the Hamiltonian

$$
\hat{H}_{1 \ldots N}=\sum_{i=1}^{N} \frac{\hat{p}_{i}^{2}}{2 m_{e}}+\frac{e^{2}}{4 \pi \varepsilon_{0}} \sum_{i<j} \frac{1}{\left|\hat{\mathbf{x}}_{i}-\hat{\mathbf{x}}_{j}\right|}+e \sum_{i=1}^{N} \varphi\left(\hat{\mathbf{x}}_{i}\right) .
$$

Here $m_{e}$ is the electron mass, $e$ is the electron charge $(e<0)$, and $\varepsilon_{0}$ is the permittivity of vacuum. The last term accounts for the interaction with the electric potential created by the ions. We now introduce the reduced density operators according to

$$
\hat{\rho}_{1 \ldots i}=n^{i} \operatorname{Tr}_{i+1 \ldots N} \hat{\rho}_{1 \ldots N} \hat{\Lambda}_{1 \ldots i},
$$

where $\operatorname{Tr}_{i+1 \ldots N}$ denotes the trace over particles $i+1$ to $N$ (i.e., integrating over the position degree of freedom and summing over the spins), $n$ is the mean density, and $\hat{\Lambda}_{1 \ldots i}$ is the antisymmetrization operator that takes an $i$-particle state and makes it completely antisymmetric [34]. We will only need to know that $\hat{\Lambda}_{12}=1-\hat{P}_{12}$ where $\hat{P}_{12}$ interchanges particle 1 and 2, i.e., $\hat{P}_{12} \psi\left(\mathbf{x}_{1}, \mathbf{x}_{2}\right)=\psi\left(\mathbf{x}_{2}, \mathbf{x}_{1}\right)$ (see, e.g., Ref. [25] for further details). The evolution for the one-particle density operator is given by

$$
i \hbar \partial_{t} \hat{\rho}_{1}=\left[\hat{h}_{1}, \hat{\rho}_{1}\right]+n \operatorname{Tr}_{2}\left[\hat{V}_{12}, \hat{\rho}_{12} \hat{\Lambda}_{12}\right],
$$

where $\quad \hat{h}_{1}=\hat{p}^{2} /\left(2 m_{e}\right) \quad$ and $\quad \hat{V}_{12}=V\left(\hat{\mathbf{x}}_{1}-\hat{\mathbf{x}}_{2}\right)=$ $e^{2} /\left(4 \pi \varepsilon_{0}\left|\hat{\mathbf{x}}_{1}-\hat{\mathbf{x}}_{2}\right|\right)$, and $\hat{\rho}_{12}$ is the two-particle density operator. The effects of two-particle correlations $\hat{g}_{12}$ can be separated out of the two-particle density operator by writing it in the form

$$
\hat{\rho}_{12}=\hat{\rho}_{1} \hat{\rho}_{2}+\hat{g}_{12}
$$

see, e.g., Ref. [35]. We are interested in the collisionless limit where a mean-field approximation will suffice. This approximation is obtained by neglecting the correlation $\hat{g}_{12}$. Utilizing this in Eq. (2), we obtain

$$
i \hbar \partial_{t} \hat{\rho}_{1}=\left[\hat{h}_{1}, \hat{\rho}_{1}\right]+\left[\bar{V}_{1}, \hat{\rho}_{1}\right]
$$


where $\bar{V}_{1}=\operatorname{Tr}_{2} \hat{V}_{12} \hat{\rho}_{2} \hat{\Lambda}_{12}$ is the Hartree-Fock potential operator. This is a closed system for the one-particle density operator.

To obtain a connection to the classical kinetic theory, we utilize the Wigner representation [36] of this equation. Using the complete set of states $|\mathbf{x}, \alpha\rangle$, where $\mathbf{x}$ is the position and $\alpha=$ 1,2 is the spin along the axis of quantization, this representation is obtained as

$$
f(\mathbf{x}, \mathbf{p}, \alpha, \beta)=\frac{n}{(2 \pi \hbar)^{3}} \int d^{3} y e^{i \mathbf{y} \cdot \mathbf{p} / \hbar} \rho\left(\mathbf{x}+\frac{\mathbf{y}}{2}, \alpha ; \mathbf{x}-\frac{\mathbf{y}}{2}, \beta\right),
$$

where $\rho(\mathbf{x}, \alpha ; \mathbf{y}, \beta)=\left\langle\mathbf{x}, \alpha\left|\hat{\rho}_{1}\right| \mathbf{y}, \beta\right\rangle$ is the density matrix. Writing Eq. (5) first in the position representation and Wigner transforming the result, we obtain

$$
\begin{array}{rl}
\partial_{t} & f(\mathbf{x}, \mathbf{p}, \alpha, \beta)+\frac{1}{m} \mathbf{p} \cdot \nabla_{x} f(\mathbf{x}, \mathbf{p}, \alpha, \beta)+\frac{i e}{\hbar} \int \frac{d^{3} y d^{3} p^{\prime}}{(2 \pi \hbar)^{3}} e^{i \mathbf{y} \cdot\left(\mathbf{p}-\mathbf{p}^{\prime}\right) / \hbar}\left[\phi\left(\mathbf{x}+\frac{\mathbf{y}}{2}\right)-\phi\left(\mathbf{x}-\frac{\mathbf{y}}{2}\right)\right] f\left(\mathbf{x}, \mathbf{p}^{\prime}, \alpha, \beta\right) \\
= & \frac{i}{\hbar(2 \pi \hbar)^{3}} \sum_{\gamma=1}^{2} \int d^{3} p^{\prime} d^{3} p^{\prime \prime} d^{3} y d^{3} r e^{i \mathbf{p} \cdot \mathbf{y} / \hbar} e^{-i \mathbf{p}^{\prime} \cdot(\mathbf{x}+\mathbf{y} / 2-\mathbf{r}) / \hbar} e^{-i \mathbf{p}^{\prime \prime} \cdot(\mathbf{r}-\mathbf{x}+\mathbf{y} / 2) / \hbar} \\
& \times\left[V\left(\mathbf{x}+\frac{\mathbf{y}}{2}-\mathbf{r}\right)-V\left(\mathbf{x}-\frac{\mathbf{y}}{2}-\mathbf{r}\right)\right] f\left(\frac{\mathbf{x}+\mathbf{r}}{2}+\frac{\mathbf{y}}{4}, \mathbf{p}^{\prime}, \alpha, \gamma\right) f\left(\frac{\mathbf{x}+\mathbf{r}}{2}-\frac{\mathbf{y}}{4}, \mathbf{p}^{\prime \prime}, \gamma, \beta\right)
\end{array}
$$

where

$$
\phi(\mathbf{x})=\frac{e n}{4 \pi \varepsilon_{0}} \sum_{\gamma=1}^{2} \int d^{3} z \frac{\rho(\mathbf{z}, \gamma ; \mathbf{z}, \gamma)}{|\mathbf{x}-\mathbf{z}|}+\varphi(\mathbf{x})
$$

is the total (mean-field and the ionic field) potential and

$$
V(\mathbf{x})=\frac{e^{2}}{4 \pi \varepsilon_{0}|\mathbf{x}|}
$$

is the Coulomb potential. The left-hand side of Eq. (7) represents the quantum Vlasov equation, while the right-hand side is the correction due to exchange effects. This term is nonlocal in phase space and nonlinear in the distribution function.

The matrix equation can be transformed into a scalar equation by taking the spin transformation [12]

$$
f(\mathbf{x}, \mathbf{p}, \mathbf{s}, t)=\frac{1}{4 \pi} \sum_{\alpha, \beta=1}^{2}\left[\delta_{\alpha, \beta}+\mathbf{s} \cdot \boldsymbol{\sigma}_{\alpha, \beta}\right] f(\mathbf{x}, \mathbf{p}, \beta, \alpha),
$$

where $\mathbf{s}$ is a vector on the unit sphere. Applying this to Eq. (7), we obtain

$$
\begin{array}{rl}
\partial_{t} & f(\mathbf{x}, \mathbf{p}, \mathbf{s})+\frac{1}{m} \mathbf{p} \cdot \nabla_{x} f(\mathbf{x}, \mathbf{p}, \mathbf{s})+\frac{i e}{\hbar} \int \frac{d^{3} y d^{3} p^{\prime}}{(2 \pi \hbar)^{3}} e^{i \mathbf{y} \cdot\left(\mathbf{p}-\mathbf{p}^{\prime}\right) / \hbar}\left[\phi\left(\mathbf{x}+\frac{\mathbf{y}}{2}\right)-\phi\left(\mathbf{x}-\frac{\mathbf{y}}{2}\right)\right] f\left(\mathbf{x}, \mathbf{p}^{\prime}, \mathbf{s}\right) \\
= & \frac{i}{\hbar} \int \frac{d^{3} p^{\prime} d^{3} p^{\prime \prime} d^{3} y d^{3} r}{(2 \pi \hbar)^{3}} e^{i \mathbf{p} \cdot \mathbf{y} / \hbar} e^{-i \mathbf{p}^{\prime} \cdot(\mathbf{x}+\mathbf{y} / 2-\mathbf{r}) / \hbar} e^{-i \mathbf{p}^{\prime \prime} \cdot(\mathbf{r}-\mathbf{x}+\mathbf{y} / 2) / \hbar} \int \frac{d^{2} s^{\prime} d^{2} s^{\prime \prime}}{8 \pi}\left[1+9 \mathbf{s}^{\prime} \cdot \mathbf{s}^{\prime \prime}+3 \mathbf{s} \cdot\left(\mathbf{s}^{\prime}+\mathbf{s}^{\prime \prime}\right)+9 i \mathbf{s} \cdot\left(\mathbf{s}^{\prime} \times \mathbf{s}^{\prime \prime}\right)\right] \\
& \times\left[V\left(\mathbf{x}+\frac{\mathbf{y}}{2}-\mathbf{r}\right)-V\left(\mathbf{x}-\frac{\mathbf{y}}{2}-\mathbf{r}\right)\right] f\left(\frac{\mathbf{x}+\mathbf{r}}{2}+\frac{\mathbf{y}}{4}, \mathbf{p}^{\prime}, \mathbf{s}^{\prime}\right) f\left(\frac{\mathbf{x}+\mathbf{r}}{2}-\frac{\mathbf{y}}{4}, \mathbf{p}^{\prime \prime}, \mathbf{s}^{\prime \prime}\right)
\end{array}
$$

where in the last term we see the exchange interaction in the Wigner form. The evolution equation (7) describes the evolution of the electrons in the mean-field (Hartree-Fock) approximation for all scale lengths. We are interested in the semiclassical limit where the potential $\phi$ and the distribution function $f$ vary on a scale $L$ much larger than the de Broglie scale length $\Lambda_{\mathrm{dB}}$ and we would like to keep only the lowest surviving correction in an expansion in $\Lambda_{\mathrm{dB}} / L$. For the potential term, the expansion is straightforward; see, for example, Ref. [5]. For the exchange term, we expand the potential and the distribution function to second order in $\mathbf{y}$ (with the assumptions that the characteristic length scale $L$ is much larger than the thermal de Broglie wavelength $\hbar / m v_{T}$, where $v_{T}$ is the thermal speed). We then perform the $\mathbf{y}$ integration and one of the momentum integrals.

Furthermore, we will for simplicity also assume that the distribution function is independent of the spin, i.e., $f(\mathbf{x}, \mathbf{p}, \mathbf{s}, t)=$ $f(\mathbf{x}, \mathbf{p}, t) /(4 \pi)$. Integrating over the spin, we obtain

$$
\begin{array}{rl}
\partial_{t} & f(\mathbf{x}, \mathbf{p}, t)+\frac{\mathbf{p}}{m} \cdot \nabla_{x} f(\mathbf{x}, \mathbf{p}, t)+e \mathbf{E}(\mathbf{x}, t) \cdot \nabla_{p} f(\mathbf{x}, \mathbf{p}, t) \\
= & \frac{1}{2} \partial_{p}^{i} \int d^{3} r d^{3} p^{\prime} e^{-i \mathbf{r} \cdot \mathbf{p}^{\prime} / \hbar}\left[\partial_{r}^{i} V(\mathbf{r})\right] f\left(\mathbf{x}-\frac{\mathbf{r}}{2}, \mathbf{p}+\frac{\mathbf{p}^{\prime}}{2}, t\right) f\left(\mathbf{x}-\frac{\mathbf{r}}{2}, \mathbf{p}-\frac{\mathbf{p}^{\prime}}{2}, t\right) \\
& -\frac{i \hbar}{8} \partial_{p}^{i} \partial_{p}^{j} \cdot \int d^{3} r d^{3} p^{\prime} e^{-i \mathbf{r} \cdot \mathbf{p}^{\prime} / \hbar}\left[\partial_{r}^{i} V(\mathbf{r})\right]\left[f\left(\mathbf{x}-\frac{\mathbf{r}}{2}, \mathbf{p}-\frac{\mathbf{p}^{\prime}}{2}, t\right)\left(\overleftarrow{\partial}_{x}^{j}-\vec{\partial}_{x}^{j}\right) f\left(\mathbf{x}-\frac{\mathbf{r}}{2}, \mathbf{p}+\frac{\mathbf{p}^{\prime}}{2}, t\right)\right],
\end{array}
$$


where $\partial_{x}^{i} \equiv \partial / \partial x_{i}$ and analogously for $\partial_{p}^{i}$, and an arrow above an operator indicates in which direction it acts. We have also used the summation convention so that a sum over indices occurring twice in a term is understood.

Equation (12) is one of the main results of this work. Unfortunately, the complexity of this equation limits the practical applicability to some extent. The key advantage is that it is derived from first principles using few assumptions besides the perturbative approach. Theories that can be applied in the regime of stronger exchange effects by necessity use assumptions and/or approximations that need validation. In some cases, justification can be done against experiments, but comparison against theory based on first principles is also much needed. Such comparisons need to be done in the regime of weak exchange effects, as it is only here that calculations based solely on first principles can be made. However, since Eq. (12) can be used as a validation for other calculation schemes (such as, e.g., density functional theory), it has relevance beyond the regime of weak exchange effects. The final section of the paper will elaborate on these issues.

\section{DAMPING OF ION-ACOUSTIC WAVES}

We now consider the effect of the exchange term on electrostatic ion-acoustic waves in a plasma. We will use Eq. (12) for the electrons and the classical Vlasov equation for ions. To obtain the dispersion relation, we assume a longitudinal oscillation $f=f_{0}(p)+f_{1}(\mathbf{p}) \exp (-i \omega t+i k z)$ and $\mathbf{E}=\hat{\mathbf{z}} E_{z} \exp (-i \omega t+i k z)$. We assume that the unperturbed electron distribution function is given by a MaxwellBoltzmann distribution [37],

$$
f_{0}(\mathbf{p})=\frac{n}{\left(2 \pi m k_{B} T_{e}\right)^{3 / 2}} \exp \left(-\frac{p_{\perp}^{2}+p_{z}^{2}}{2 m k_{B} T_{e}}\right) .
$$

Furthermore, assuming that the exchange terms are a small correction to the distribution function, we may calculate it by inserting the lowest-order solution for $f_{1}$, i.e., $f_{1}=$ $-i e E_{z} /\left(\omega-k p_{z} / m\right) \partial_{p_{z}} f_{0}$, in the integrand. Introducing spatial spherical coordinates, it is possible to solve all spatial integrals in Eq. (12). Next, the integrand is expanded in terms of $\hbar$. The lowest-order term in the first integral vanishes due to symmetry and we keep only the two first-order terms. In the second integral, we already have an additional $\hbar$, meaning that we only retain the lowest-order term. Finally, it is possible to solve the $p_{z}^{\prime}$ and $\varphi_{p}^{\prime}$ integrals. The remaining integrals are solved numerically and doing so gives a solution for $f_{1}$ in the linear regime. Now, from the classical dispersion relation, we have

$$
\omega \approx\left(\omega_{p I} / \omega_{p e}\right) k v_{T e} \equiv \alpha k v_{T e},
$$

where $v_{T e}=\sqrt{k_{B} T_{e} / m_{e}}$ is the electron thermal velocity and $\omega_{p}$ denotes the plasma frequency. The dispersion relation is then given by

$$
0=1+\frac{\omega_{p e}^{2}}{k^{2} v_{T e}^{2}}-\frac{\omega_{p I}^{2}}{\omega^{2}}-\frac{\hbar^{2} \omega_{p e}^{4}}{4 \pi m^{2} k^{2} v_{T e}^{6}} \int d v \frac{e^{-v^{2}}}{(\alpha-v)^{2}} \int d u\left[\frac{v+u}{\alpha-(v+u)}\right]\left[\left(u^{2}-\frac{u}{\alpha-v}-\frac{1}{2}\right) \operatorname{Ei}\left(-u^{2}\right)+e^{-u^{2}}\right]
$$

where the first three terms give the classical dispersion relation for an ion-acoustic wave (Ei denotes the exponential integral). Solving these integrals numerically gives the approximate dispersion relation,

$0 \approx 1+\frac{\omega_{p e}^{2}}{k^{2} v_{T e}^{2}}\left(1+\frac{2 i \gamma_{\mathrm{cl}}}{k c_{s}}\right)-\frac{\omega_{p I}^{2}}{\omega^{2}}-\frac{\hbar^{2} \omega_{p e}^{4}}{m^{2} k^{2} v_{T e}^{6}}(0.8+0.05 i)$

which, in the quasineutral limit $\omega_{p e}^{2} \gg k^{2} v_{T e}^{2}$, can be written

$$
\omega=k c_{s}\left(1+0.8 \frac{\hbar^{2} \omega_{p e}^{2}}{m^{2} v_{T e}^{4}}\right)-i \gamma_{\mathrm{cl}}\left(1-3 \frac{\hbar^{2} \omega_{p e}^{2}}{m^{2} v_{T e}^{4}}\right)
$$

where $c_{s}=\left(m_{e} / m_{i}\right)^{1 / 2} v_{T e}$ is the classical ion-acoustic velocity and we have introduced the classical electron Landau damping, $\gamma_{\mathrm{cl}}=k c_{s} \sqrt{\pi / 8} \sqrt{m_{e} / m_{i}}$, in the cold ion limit [38].

\section{APPLICABILITY OF MODEL AND RESULTS}

In deriving our model given by Eq. (12) and the result (16), a series of approximations have been made and here we give a brief recapitulation of these together with a discussion of their implications for the applicability of the results.

In order to obtain a closure relation for the BogoliubovBorn-Green-Kirkwood-Yvon (BBGKY) hierarchy, we have neglected particle-particle correlations in Eq. (4), which means disregarding the collisional influence. This approximation is known to be particle and energy conserving [25]. A general motivation for neglecting the collisions in our calculations is that the effect of these have been much studied; see, e.g., [39-44]. Furthermore, since we are utilizing a perturbative method, the two effects can be studied separately and added together afterwards provided the collisions also are weak. The relative magnitude of the collisional influence scales as (see, e.g., [39]) $\left(E_{p} / E_{k}\right)^{3 / 2}$, where $E_{p}=q^{2} n_{0}^{1 / 3} / \varepsilon_{0}$ is the characteristic potential energy between nearest neighbors and $E_{k}=k_{B} T_{e}=m_{e} v_{T e}^{2} / 2$ gives the average kinetic energy of an electron. To check that the magnitude of exchange corrections is not necessarily negligible compared to collisions, we rewrite the parameter of the previous section as $\left(\hbar \omega_{p e} / m v_{T e}^{2}\right)^{2} \sim$ $\left(E_{p} / E_{k}\right)\left(E_{F} / E_{k}\right)$ [5], where $E_{F}$ is the Fermi energy. Thus we find that the ratio $R$ of exchange effects over collisions scales as

$$
R \sim \frac{E_{F}}{E_{k}^{1 / 2} E_{p}^{1 / 2}} .
$$

From this, we find that we may indeed find a regime where exchange effects dominate over collisions by choosing a sufficiently high density so that $E_{F} \gg E_{p}$ and then keep the temperature relatively modest such that $E_{k}$ is not too much larger than $E_{F}$. 
In our calculations, we furthermore used a MaxwellBoltzmann background distribution function instead of a Fermi-Dirac distribution. This was done mainly due to technical reasons, since it facilitates the solutions of some of the integrals, but is a good approximation as long as $E_{F} / E_{k}$ is small. We have also used the long-scale length limit, which should be valid given that the de Broglie wavelength of the particles is short enough compare to the scale lengths we are interested in.

\section{DISCUSSION}

From Eq. (16), we note that the effective ion-acoustic velocity is increased, whereas the damping due to waveparticle interaction is decreased due to the exchange effect. As seen from (16), the relative magnitude of both of these effects is of the order of $H^{2}$, where $H=\hbar \omega_{p e} / m v_{T e}^{2}$. As is shown in, e.g., Ref. [5], plotting the line $H=1$ in a log-log density temperature diagram divides the parameter space in a classical regime $(H \ll 1)$ and a strong quantum regime $(H \gtrsim$ 1). However, such plots are typically performed in order to illustrate the relative importance of particle dispersive effects. Within a kinetic formalism, particle dispersion is described by the terms with higher-order momentum and spatial derivatives in the Wigner equation [5]. For such terms to be important, in addition to the parameter $H$ not being too small, we also require the scale lengths under study to be short. Specifically, we need the scale lengths to approach the thermal de Broglie wavelength or shorter. Thus if we exclude the regime of short scale lengths, as we have done here, the quantum effect of particle dispersion is guaranteed to be of little significance. By contrast, we see that exchange effects may very well affect the long-scale behavior of the low-frequency density dynamics. Of particular interest is the change in the damping term. By approaching the regime $H \sim 1$, Eq. (16) suggests that we may more or less completely suppress Landau damping of ion-acoustic waves. Physically this makes sense, as classically the particles that are resonantly accelerated for a long time are rather well localized in phase space, which is then counteracted by the exchange terms. Strictly, the regime $H \sim 1$ does not fit into the perturbation scheme that we have applied here, but qualitatively we still expect this result to be valid.

Plasmas where exchange effects can be important occur in, e.g., laser-plasma interaction experiments on solid targets, such as in inertial confinement fusion schemes. After the compression phase, but before the main part of the heating has occurred we may have a plasma density of the order of $10^{32} \mathrm{~m}^{-3}$ and a temperature of $T=4 \times 10^{6} \mathrm{~K}$, in which case the Debye length and the de Broglie length are comparable and we have $\hbar \omega_{p} / k_{B} T \simeq 1$. For these parameters, the plasma is partially degenerate, and the Fermi temperature $T_{F}$ and the thermodynamic temperature are comparable. Since we have considered the case $\hbar \omega_{p} / k_{B} T \ll 1$, however, a substantially higher value of the electron temperature and the ordering $T_{F} \ll T$ is consistent with our calculations.

An important result from this study is the general expression for the exchange term, as given by Eq. (12). This term can describe exchange modification of any type of processes, e.g., altering the coefficients for three-wave interaction $[45,46]$, adjusting the Zakharov equations $[47,48]$, or modifying non- linear wave-particle interaction processes $[49,50]$. The main restriction is due to the assumption of electrostatic fields. The complexity of the exchange interaction term in Eq. (12) in practice forces one to do perturbative calculations. Since the present formalism captures the full effect of a distribution function which may be far from equilibrium, it provides a valuable opportunity to evaluate approaches that rely on other types of approximations. Specifically, in time-dependent density functional theory (TDDFT) [51], the properties of the system are derived from the electron density only (or are at least limited to macroscopic quantities), in which case the full dependence on the detailed momentum distribution is disregarded. Due to the complexity of many nonlinear plasma systems, such a drastic simplification may be needed, but at the same time is it essential that the accuracy of the approach can be evaluated. Results from DFT calculations have been used to describe electrostatic waves in plasmas; see, e.g., Eq. (6) of Ref. [52], where the further approximation of the adiabatic local density approximation (ALDA) has been used. See also Ref. [53] where the exchange effects on nonlinear ion-acoustic waves have been studied. However, a difference with our case is that the Fermi temperature was assumed to be higher than the plasma temperature in these papers. In a very rough sense, the previous results agree with ours, as the relative importance of the exchange term scales as $\left(\hbar \omega_{p} / E_{K}\right)^{2}$ in both cases, noting that the characteristic kinetic energy $E_{k}$ is the thermal energy $k_{B} T$ in our case and the Fermi energy $k_{B} T_{F}$ in the case of Ref. [52]. However, in our case, the phase velocity of the ion-acoustic waves is increased due to the exchange interaction, whereas based on Eq. (6) of Ref. [52], the phase velocity is decreased. Still, the interpretation of this fact can be debated. One possibility is that the approximation of ALDA to evaluate the exchange potential is too restrictive to capture the ion-acoustic dynamics accurately. Another possibility is that the results are indeed sensitive to the ordering of $T$ and $T_{F}$, such that the sign of the exchange effect is reversed when the ordering is changed. Regardless of this, is is clear that DFT calculations in general cannot capture the effects of wave-particle interaction, which is responsible for the wave damping in our case.

\section{ACKNOWLEDGMENTS}

This research was supported by the Swedish Research Council Grants No. 2010-3727 and No. 2012-5644, and the European Research Council Grant No. 204059-QPQV and “ATOMAG” ERC-2009-AdG-20090325 Grant No. 247452.

\section{APPENDIX: LONG-SCALE LENGTH LIMIT OF THE EXCHANGE TERM}

The calculations leading from Eqs. (11) to (12) are somewhat complicated and here we show the steps in more detail. Since the long-scale length limit of the left-hand side of (11) is already known (see, e.g., [5]), we focus solely on the exchange term on the right-hand side. The first step is to assume a spinindependent distribution function and thereby solve the spin integrals which are then trivial. We then expand the distribution function and the potential $V$ to second order in $\mathbf{y}$ and use the 
identity $y_{j} \exp (i \mathbf{p} \cdot \mathbf{y} / \hbar)=-i \hbar \partial_{p}^{j} \exp (i \mathbf{p} \cdot \mathbf{y} / \hbar)$ to get

$$
\begin{aligned}
I= & -\int \frac{d p^{\prime} d p^{\prime \prime} d y d z}{2(2 \pi \hbar)^{3}} e^{i \mathbf{p} \cdot \mathbf{y} \hbar} e^{-i \mathbf{p}^{\prime} \cdot(\mathbf{x}+\mathbf{y} / 2-\mathbf{z}) / \hbar} e^{-i \mathbf{p}^{\prime \prime} \cdot(\mathbf{z}-\mathbf{x}+\mathbf{y} / 2) / \hbar}\left(-i \hbar \overleftarrow{\partial}_{p}^{i}\right)\left[\partial_{x}^{i} V(\mathbf{x}-\mathbf{z})\right] \\
& \times\left[f\left(\frac{\mathbf{x}+\mathbf{z}}{2}, \mathbf{p}^{\prime}\right) f\left(\frac{\mathbf{x}+\mathbf{z}}{2}, \mathbf{p}^{\prime \prime}\right)-\frac{i \hbar}{4} \overleftarrow{\partial}_{p}^{j} f\left(\frac{\mathbf{x}+\mathbf{z}}{2}, \mathbf{p}^{\prime \prime}\right) \partial_{\frac{x+z}{2}}^{j} f\left(\frac{\mathbf{x}+\mathbf{z}}{2}, \mathbf{p}^{\prime}\right)\right. \\
& \left.+\frac{i \hbar}{4} \overleftarrow{\partial}_{p}^{j} f\left(\frac{\mathbf{x}+\mathbf{z}}{2}, \mathbf{p}^{\prime}\right) \partial_{\frac{x+z}{2}}^{j} f\left(\frac{\mathbf{x}+\mathbf{z}}{2}, \mathbf{p}^{\prime \prime}\right)\right],
\end{aligned}
$$

where the arrow above the derivative signifies the direction in which the derivative acts. The next step is to perform the integration over $\mathbf{y}$, which is now straightforward as we only have

$$
\begin{aligned}
& i \hbar \partial_{p}^{i} \int \frac{d p^{\prime} d p^{\prime \prime} d z}{2} \delta\left(\mathbf{p}-\frac{\mathbf{p}^{\prime}+\mathbf{p}^{\prime \prime}}{2}\right) e^{-i\left(\mathbf{p}^{\prime}-\mathbf{p}^{\prime \prime}\right) \cdot(\mathbf{x}-\mathbf{z}) / \hbar}\left[\partial_{x}^{i} V(\mathbf{x}-\mathbf{z})\right]\left[f\left(\frac{\mathbf{x}+\mathbf{z}}{2}, \mathbf{p}^{\prime}\right) f\left(\frac{\mathbf{x}+\mathbf{z}}{2}, \mathbf{p}^{\prime \prime}\right)\right. \\
& \left.\quad-\frac{i \hbar}{4} \overleftarrow{\partial}_{p}^{j} f\left(\frac{\mathbf{x}+\mathbf{z}}{2}, \mathbf{p}^{\prime \prime}\right) \partial_{\frac{x+z}{2}}^{j} f\left(\frac{\mathbf{x}+\mathbf{z}}{2}, \mathbf{p}^{\prime}\right)+\frac{i \hbar}{4} \overleftarrow{\partial}_{p}^{j} f\left(\frac{\mathbf{x}+\mathbf{z}}{2}, \mathbf{p}^{\prime}\right) \partial_{\frac{x+z}{2}}^{j} f\left(\frac{\mathbf{x}+\mathbf{z}}{2}, \mathbf{p}^{\prime \prime}\right)\right] .
\end{aligned}
$$

In order to keep the result more symmetric, we make the substitution

$$
\begin{aligned}
& \mathbf{p}_{1}=\mathbf{p}^{\prime}-\mathbf{p}^{\prime \prime}, \\
& \mathbf{p}_{2}=\frac{\mathbf{p}^{\prime}+\mathbf{p}^{\prime \prime}}{2},
\end{aligned}
$$

and then perform the integration over $\mathbf{p}_{2}$, which is easy due to the $\delta$ function. The result is

$$
\begin{aligned}
& i \hbar \partial_{p}^{i} \int \frac{d p_{1} d z}{2} e^{-i \mathbf{p}_{1} \cdot(\mathbf{x}-\mathbf{z}) / \hbar}\left[\partial_{x}^{i} V(\mathbf{x}-\mathbf{z})\right]\left\{f\left(\frac{\mathbf{x}+\mathbf{z}}{2}, \mathbf{p}+\frac{\mathbf{p}_{1}}{2}\right) f\left(\frac{\mathbf{x}+\mathbf{z}}{2}, \mathbf{p}-\frac{\mathbf{p}_{1}}{2}\right)-\frac{i \hbar}{4}\right. \\
& \left.\quad \times \partial_{p}^{j}\left[f\left(\frac{\mathbf{x}+\mathbf{z}}{2}, \mathbf{p}-\frac{\mathbf{p}_{1}}{2}\right) \partial_{\frac{x+z}{2}}^{j} f\left(\frac{\mathbf{x}+\mathbf{z}}{2}, \mathbf{p}+\frac{\mathbf{p}_{1}}{2}\right)-f\left(\frac{\mathbf{x}+\mathbf{z}}{2}, \mathbf{p}+\frac{\mathbf{p}_{1}}{2}\right) \partial_{\frac{x+z}{2}}^{j} f\left(\frac{\mathbf{x}+\mathbf{z}}{2}, \mathbf{p}-\frac{\mathbf{p}_{1}}{2}\right)\right]\right\},
\end{aligned}
$$

where we have also factored out the momentum derivative on the last two terms. Finally, we make the variable substitution $\mathbf{z} \rightarrow \mathbf{x}-\mathbf{z}$ and obtain the required result:

$$
\begin{aligned}
= & \frac{i \hbar}{2} \partial_{p}^{i} \int d p_{1} d z e^{-i \mathbf{p}_{1} \cdot \mathbf{z} / \hbar}\left[\partial_{z}^{i} V(\mathbf{z})\right] f\left(\mathbf{x}-\frac{\mathbf{z}}{2}, \mathbf{p}+\frac{\mathbf{p}_{1}}{2}\right) f\left(\mathbf{x}-\frac{\mathbf{z}}{2}, \mathbf{p}-\frac{\mathbf{p}_{1}}{2}\right)-\frac{\hbar^{2}}{8} \partial_{p}^{i} \partial_{p}^{j} \int d p_{1} d z e^{-i \mathbf{p}_{1} \cdot \mathbf{z} / \hbar}\left[\partial_{z}^{i} V(\mathbf{z})\right] \\
& \times\left[f\left(\mathbf{x}-\frac{\mathbf{z}}{2}, \mathbf{p}-\frac{\mathbf{p}_{1}}{2}\right) \partial_{x}^{j} f\left(\mathbf{x}-\frac{\mathbf{z}}{2}, \mathbf{p}+\frac{\mathbf{p}_{1}}{2}\right)-f\left(\mathbf{x}-\frac{\mathbf{z}}{2}, \mathbf{p}+\frac{\mathbf{p}_{1}}{2}\right) \partial_{x}^{j} f\left(\mathbf{x}-\frac{\mathbf{z}}{2}, \mathbf{p}-\frac{\mathbf{p}_{1}}{2}\right)\right] .
\end{aligned}
$$

[1] G. Mourou, T. Tajima, and S. V. Bulanov, Rev. Mod. Phys. 78, 309 (2006).

[2] M. Marklund and P. K. Shukla, Rev. Mod. Phys. 78, 591 (2006).

[3] S. H. Glenzer and R. Redmer, Rev. Mod. Phys. 81, 1625 (2009).

[4] A. Di Piazza, C. Müller, K. Z. Hatsagortsyan, and C. H. Keitel, Rev. Mod. Phys. 84, 1177 (2012).

[5] G. Manfredi, in Topics in Kinetic Theory, edited by. T. Passot, C. Sulem, and P.-L. Sulem, Fields Institute Communications, Vol. 46 (American Math. Soc., Providence, 2005), pp. 263-288.

[6] F. Haas, Quantum Plasmas, A Hydrodynamics Approach (Springer, New York, 2011).

[7] P. K. Shukla and B. Eliasson, Rev. Mod. Phys. 83, 885 (2011).

[8] P. Burt and H. Wahlquist, Phys. Rev. 125, 1785 (1962).

[9] S. Cowley, R. Kulsrud, and E. Valeo, Phys. Fluids 29, 430 (1986).

[10] G. Brodin, M. Marklund, J. Zamanian, A. Ericsson, and P. L. Mana, Phys. Rev. Lett. 101, 245002 (2008).

[11] O. Morandi, P. Hervieux, and G. Manfredi, New J. Phys. 11, 073010 (2009).

[12] J. Zamanian, M. Marklund, and G. Brodin, New J. Phys. 12, 043019 (2010).
[13] J. Zamanian, M. Stefan, M. Marklund, and G. Brodin, Phys. Plasmas 17, 102109 (2010).

[14] N. Shukla, P. Shukla, B. Eliasson, and L. Stenflo, Phys. Lett. A 374, 1749 (2010).

[15] G. Brodin, A. P. Misra, and M. Marklund, Phys. Rev. Lett. 105, 105004 (2010).

[16] M. Stefan, J. Zamanian, G. Brodin, A. P. Misra, and M. Marklund, Phys. Rev. E 83, 036410 (2011).

[17] G. Brodin, M. Marklund, and G. Manfredi, Phys. Rev. Lett. 100, 175001 (2008).

[18] D. Shaikh and P. K. Shukla, Phys. Rev. Lett. 99, 125002 (2007).

[19] G. Manfredi and P.-A. Hervieux, Phys. Rev. B 72, 155421 (2005).

[20] P. Hohenberg and W. Kohn, Phys. Rev. 136, B864 (1964).

[21] W. Kohn and L. Sham, Phys. Rev. 385, A1133 (1965).

[22] O. Von Roos, Phys. Rev. 119, 1174 (1960).

[23] O. Von Roos and J. Zmuidzinas, Phys. Rev. 121, 941 (1961).

[24] Y. L. Klimontovich and W. D. Kraeft, Theor. Math. Phys. 19, 574 (1974). 
[25] M. Bonitz, Quantum Kinetic Theory (Teubner, Stuttgart, 1998).

[26] F. Brosens and J. T. Devreese, Phys. Rev. B 29, 543 (1984).

[27] H. Nachtegaele, F. Brosens, and J. T. Devreese, Phys. Rev. B 28, 6064 (1983).

[28] Y. Ma, S. Mao, and J. Xue, Phys. Plasmas 18, 102108 (2011).

[29] F. Haas, L. G. Garcia, J. Goedert, and G. Manfredi, Phys. Plasmas 10, 3858 (2003).

[30] A. P. Misra and A. R. Chowdhury, Phys. Plasmas 13, 072305 (2006).

[31] S. Mahmood and A. Mushtaq, Phys. Lett. A 372, 3467 (2008).

[32] A. Mushtaq and D. B. Melrose, Phys. Plasmas 16, 102110 (2009).

[33] T. S. Gill, A. S. Bains, and C. Bedi, J. Phys.: Conf. Ser. 208 012040 (2010).

[34] D. B. Boercker and J. W. Dufty, Ann. Phys. 119, 43 (1979).

[35] S. J. Wang and W. Cassing, Ann. Phys. 159, 328 (1985).

[36] E. Wigner, Phys. Rev. 40, 749 (1932).

[37] For dense systems, where exchange effects can be of importance, the ratio of the Fermi temperature and the thermodynamic temperature can vary considerably, including both the fully degenerate and the completely nondegenerate plasma. Here we focus on the latter case, corresponding to, for example, the later stages in the inertial confinement fusion regime, not too far from ignition. Furthermore, expanding the Fermi-Dirac distribution in the classical limit (i.e., when the chemical potential $\mu \rightarrow-\infty$ ) gives a correction proportional to $\hbar^{3} n / m^{3} v_{T}^{3} \ll \Lambda_{\mathrm{dB}}^{3} / L^{3} \ll 1$.

[38] T. J. M. Boyd and J. J. Sanderson, The Physics of Plasmas (Cambridge University Press, Cambridge, 2003).

[39] R. Cohen, L. J. Spitzer, and P. Routly, Phys. Rev. 263, 230 (1950).

[40] L. Spitzer, Jr. and R. Härm, Phys. Rev. 89, 977 (1953).

[41] A. Lenard, Ann. Phys. (NY) 10, 390 (1960).

[42] R. Balescu, Phys. Fluids 3, 52 (1960).

[43] E. M. Epperlein, R. W. Short, and A. Simon, Phys. Rev. Lett. 69, 1765 (1992).

[44] E. M. Epperlein, Phys. Plasmas 1, 109 (1994).

[45] G. Brodin and L. Stenflo, J. Plasma Phys. 39, 277 (1988).

[46] L. Stenflo, J. Plasma Phys. 4, 585 (1970).

[47] V. E. Zakharov, Zh. Eksp. Teor. Fiz. 62, 1745 (1972) [Sov. Phys. JETP 35, 908 (1972)].

[48] V. I. Karpman, Phys. Plasmas 5, 932 (1998).

[49] G. Brodin, Phys. Rev. Lett. 78, 1263 (1997).

[50] G. Manfredi, Phys. Rev. Lett. 79, 2815 (1997).

[51] M. A. L. Marques et al., Time Dependent Density Functional Theory, Lecture Notes in Physics Vol. 706 (Springer, BerlinHeidelberg, 2006).

[52] N. Crouseilles, P.-A. Hervieux, and G. Manfredi, Phys. Rev. B 78, 155412 (2008).

[53] K. Ourabah and M. Tribeche, Phys. Rev. E 88, 045101 (2013). 